\title{
Promoção de saúde e marketing social
}

Paula Broeiro*

$\mathrm{N}$ o final de 2014 decorreu a última campanha da Fundação Portuguesa "Comunidade contra a SIDA" e o Congresso Nacional VIH, Doenças Infecciosas e Microbiologia Clínica. A ênfase, nos órgãos de comunicação social, foi dada aos testes de rastreio.

Hoje, o novo tratamento da hepatite C (Sofosbuvir) inunda os telejornais.

A aparente contradição na ordem natural do investimento em saúde leva-nos a questionar porque se centram as notícias de divulgação pública no diagnóstico e na terapêutica. Onde está o investimento na educação e promoção de saúde e, consequentemente, na adoção de comportamentos saudáveis? Esta reflexão fez-nos regressar aos conceitos de promoção de saúde/sentido de coerência e às questões da comunicação em saúde/marketing social.

Com a Carta de Otawa, a saúde passou a ser vista como um conceito positivo que acentua a importância dos recursos sociais e pessoais, decorrentes estes da garantia das condições básicas e recursos fundamentais como: paz, abrigo, educação, alimentação, entre outros. ${ }^{1}$ A promoção de saúde é, assim, o processo que visa aumentar a capacidade dos indivíduos e das comunidades para controlarem a sua saúde, no sentido de a melhorar. ${ }^{1}$ A saúde é, em si, um bem civilizacional que torna evidente as desigualdades atribuíveis ao "gradiente social". Dos determinantes sociais da saúde, a educação é a que melhor se relaciona com o maior grau de coesão social, melhor saúde, bem-estar e comportamentos saudáveis na vida adulta. ${ }^{2}$ Há evidência de que a educação pode ajudar a reduzir a incidência de doenças crónicas e a melhorar a gestão pessoal de doença e que pode ajudar a diminuir a prevalência de doenças sexualmente transmissíveis. ${ }^{2}$

Tendo subjacente a essência da Carta de Ottawa, Antonovsky introduziu o conceito de salutogénese (pensar o que gera saúde, ao invés de pensar apenas o que gera

*Directora da Revista Portuguesa de Medicina Geral e Familiar doença) e Sentido de Coerência. ${ }^{3-4}$ Defende que o sentido de coerência é a capacidade de cada pessoa compreender as situações de stress e de mobilizar recursos pessoais, sociais e de saúde para as ultrapassar e que a autoconfiança resultante se reflete na forma como cada um pensa, é e age. Neste modelo conceptual, a promoção da saúde não se limita a comportamentos relacionados com a saúde e não se centra apenas no comportamento individual, mas também na forma como a sociedade se organiza. ${ }^{3}$ De acordo com Antonovsky, a metáfora "a saúde no rio da vida" (Figura 1) sustenta que não basta evitar o stress e que, em vez disso, as pessoas deviam ser autossuficientes, "aprender a nadar". ${ }^{3-4}$ Mas também que é necessário implementar os princípios da salutogénese e sentido de coerência em todas as políticas e incluí-los no processo de aprendizagem e de desenvolvimento na escola. ${ }^{3}$

Intervenções baseadas na escola, para melhorar o conhecimento sexual e de doenças sexualmente transmissíveis relacionadas com comportamentos, bem como programas antitabágicos e de prevenção da toxicodependência, tendem a mostrar efeitos positivos em todos os países, apesar de a evidência do seu impacto na saúde a longo prazo continuar a ser limitada. ${ }^{2}$ As intervenções relacionadas com a mudança de hábitos devem ser, social e culturalmente, influenciadas, isto porque os comportamentos são determinados pelo que é aceite ou incentivado socialmente. ${ }^{2-5}$ Intervenções que visam gerar uma mudança de estilos de vida entre os jovens e as crianças conduzem a uma mudança de valores sociais, devendo, por isso, usar recursos educacionais que incentivem efetivamente a necessária transformação cultural. ${ }^{2}$

O sucesso da adoção coletiva de comportamentos saudáveis precisa que os decisores entendam o problema como uma necessidade que requer uma abordagem holística, em que a Comunicação em Saúde e o Marketing Social podem ter um papel relevante. O marketing social é amplamente utilizado para influenciar o comportamento de saúde e aplica uma vasta gama de 


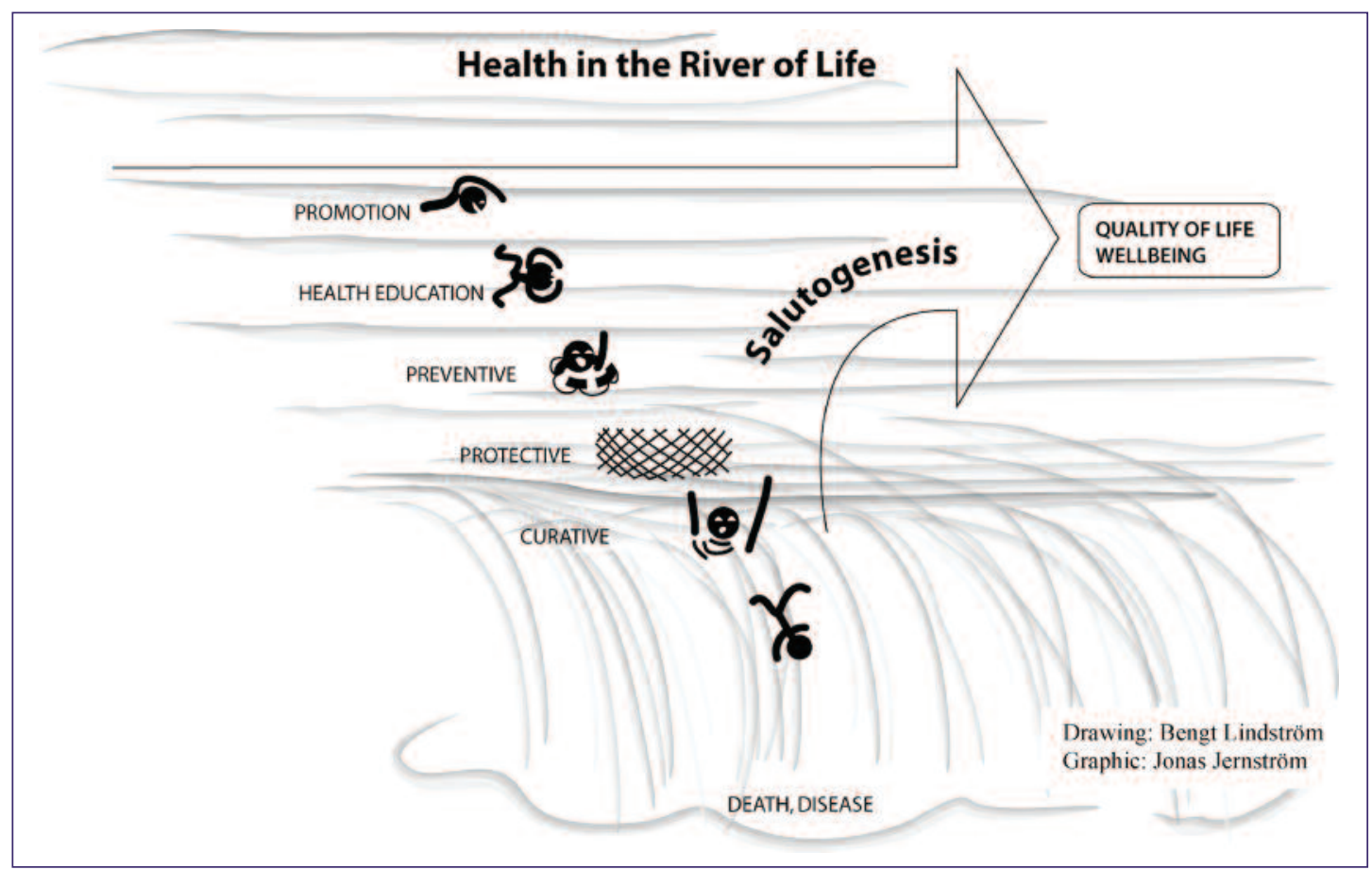

Figura 1. A saúde no rio da vida. ${ }^{3 *}$

*Figura publicada com permissão dos autores.

estratégias de comunicação; ${ }^{6}$ utiliza estratégias de marketing comercial à promoção da saúde pública, é eficaz a nível populacional e pode contribuir para a eficácia das intervenções; ${ }^{6}$ visa alterar o comportamento da população-alvo e, para tal, avalia os fatores que fundamentam a recetividade da mensagem, tal como a credibilidade e simpatia do argumento e utiliza a teoria comportamental. ${ }^{6}$ Este marketing, que visa mudar o comportamento de saúde, encontra concorrência, tendo como desafio o ser capaz de competir com os anunciantes de produtos com orçamentos superiores e diversidade de formas de chegar aos consumidores. ${ }^{6}$

Um estudo de 17 campanhas de saúde europeias recentes sobre uma variedade de tópicos, incluindo a promoção de testes para VIH, demonstrou que os comportamentos a necessitar de serem alterados uma ou, apenas, algumas vezes são mais fáceis de promover do que aqueles que devem ser repetidos e mantidos ao longo do tempo. ${ }^{6}$ As campanhas de comunicação em saúde devem integrar mensagens que, direta ou indiretamente, informem e, consequentemente, influenciem a alteração e manutenção de comportamentos saudáveis. ${ }^{7}$ Existe evidência de que campanhas de comunicação em saúde, por si só, aumentam a consciencialização e o conhecimento e, assim, podem contribuir para mudanças de atitudes e intenções comportamentais. ${ }^{7}$ As campanhas de comunicação de saúde pública complementadas com outras estratégias (e.g., os serviços de rastreio) são mais propensas a produzir mudanças positivas de comportamentos relacionados com a saúde do que as campanhas de comunicação em saúde isoladas.?

Sabemos que as pessoas tomam, ou não, decisões saudáveis dentro do contexto do ambiente social e cultural em que vivem. Se as pessoas não dispõem de um ambiente seguro para o exercício físico provavelmente não o praticarão. Se os preservativos não são, socialmente, aceitáveis ou acessíveis não serão utilizados. A teoria eco-social é um quadro importante que oferece insight sobre como e porque esses comportamentos ocorrem. Esta teoria identifica múltiplos níveis de influência (intrapessoais, sociais, ambientais e institucionais) e emprega uma variedade de disciplinas e perspetivas, num esforço de compreensão e abordagem de 
problemas de saúde pública complexos. ${ }^{8} \mathrm{O}$ axioma fundamental no marketing social é a noção de troca voluntária: que os indivíduos adotam produtos, ideias e comportamentos esperando benefícios. As abordagens combinadas de marketing social e teoria eco-social com foco em pessoas e lugares pode resultar em mudanças de comportamento permanentes. ${ }^{8}$

A infeção VIH-SIDA é paradigmática do papel dos determinantes sociais de saúde, verificando-se que em Portugal, desde 2000, se regista uma frequência crescente de casos entre imigrantes e aumento da proporção de novos casos na população com idade superior a 49 anos. ${ }^{9}$ Apesar disso, verifica-se uma tendência de decréscimo do número de novos casos e um declínio consistente da proporção de casos de SIDA relativamente ao total de novos casos, tal como noutros países ocidentais..$^{9} \mathrm{O}$ sucesso das campanhas relacionadas com os direitos humanos, do UNAIDS: "Zero novas infecções", "Zero mortes relacionadas com a SIDA" e "Zero Discriminação", ${ }^{10}$ depende da coerência, persistência e continuidade da prossecução destes objetivos.

Em Portugal, o marketing social em saúde parece ter pouca expressão, não se antecipando aos fenómenos sociológicos da saúde. A complexidade deste fenómeno requer que, em todos os problemas relacionados com o comportamento, não se abandone a promoção de saúde (e.g., Zero novas infecções), antes se integre em programas de educação e em campanhas de marketing social ciclicamente renovadas e concorrenciais com as indústrias, farmacêutica e de dispositivos mé- dicos (e.g., testes de diagnóstico) e se apele ao sentido de coerência individual e coletiva.

\section{REFERÊNCIAS BIBLIOGRÁFICAS}

1. World Health Organization. The OTTAWA charter for health promotion [Internet]. Geneva: WHO; 1986. Available from: http://www.who.int/healthpromotion/conferences/previous/ottawa/en/

2. World Health Organization. The economics of social determinants of health and health inequalities. Geneva: WHO; 2013. ISBN 9789241548625

3. Eriksson M, Lindström B. A salutogenic interpretation of the Ottawa Charter. Health Promot Int. 2008;23(2):190-9.

4. Antonovsky A. The salutogenic model as a theory to guide health promotion. Health Promot Int. 1996;11(1):11-8.

5. Yaphe J. Culture and health: why we need medical anthropology in family medicine in Portugal. Rev Port Med Geral Fam. 2014;30(6):354-5.

6. Evans WD. How social marketing works in health care. BMJ. 2006;332(7551):1207-10.

7. Robinson MN, Tansil KA, Elder RW, Soler RE, Labre MP, Mercer SL, et al. Mass media health communication campaigns combined with healthrelated product distribution: a community guide systematic review. Am J Prev Med. 2014;47(3):360-71.

8. Daniel KL, Bernhardt JM, Ero lu D. Social marketing and health communication: from people to places. Am J Public Health. 2009;99(12):2120-2.

9. Diniz A, Duarte R, Caldeira C, Bettencourt J, Gomes M, Oliveira O, et al. Portugal, infeção VIH, SIDA e tuberculose em números - 2014. Lisboa: Direção-Geral da Saúde; 2014.

10. UNAIDS. Getting to zero: 2011-2015 Strategy Joint United Nations Programme on HIV/AIDS (UNAIDS). Washington: World Health Organization; 2011. ISBN 97892917388305

\section{ENDEREÇO PARA CORRESPONDÊNCIA}

director@rpmgf.pt 